Clio. Femmes, Genre, Histoire

1 | 1995

Résistances et Libérations France 1940-1945

\title{
Une femme nouvelle pour une France nouvelle?
}

\section{Claire DUCHEN}

\section{OpenEdition}

Journals

Édition électronique

URL : https://journals.openedition.org/clio/520

DOI : 10.4000/clio.520

ISSN : 1777-5299

Éditeur

Belin

Édition imprimée

Date de publication : 1 avril 1995

ISSN : 1252-7017

Référence électronique

Claire DUCHEN, « Une femme nouvelle pour une France nouvelle? », Clio. Femmes, Genre, Histoire [En ligne], 1 | 1995, mis en ligne le 01 janvier 2005, consulté le 23 avril 2022. URL : http://

journals.openedition.org/clio/520 ; DOI : https://doi.org/10.4000/clio.520

Ce document a été généré automatiquement le 23 avril 2022

Tous droits réservés 


\title{
Une femme nouvelle pour une France nouvelle?
}

\author{
Claire DUCHEN
}

1 «Il a surgi de ce chaos une femme nouvelle» proclame Mathilde Péri, déléguée communiste à l'Assemblée consultative provisoire le 15 mai 1945. Pour les femmes, à l'euphorie de la Libération s'ajoute l'optimisme pour l'avenir: tous les changements semblent possibles. Les femmes françaises reçoivent le droit de vote et d'éligibilité en 1944 et se permettent d'espérer que les autres droits en découleront.

2 Une femme nouvelle, donc, dans une France nouvelle ? Comme tant d'autres, à cette époque, Mathilde Péri évoque le rôle social et économique que la femme nouvelle va jouer dans la France nouvelle; mais ce discours de la femme nouvelle, tant répété dans les années de la Libération, est-il porteur de changements réels dans la vie des femmes, ou fait-il plutôt partie d'une stratégie rhétorique pour mettre une distance entre l'Occupation et l'après-guerre - distance selon laquelle tout est obligatoirement nouveau ? Une femme nouvelle a-t-elle réellement surgi de la guerre ? Le suffrage va-til entraîner la transformation des rôles féminins dans la nation, et, également, dans la vie privée?

De fait, les changements profonds, tant des rôles féminins que du discours sur ces rôles, sont lents à entrer dans la pratique. Considérer les changements survenus dans la vie des femmes exige un regard sur la période de l'après-Libération, car les années de la Libération sont toujours celles de privations et de difficultés dans la vie quotidienne, de rupture dans la vie personnelle. C'est à partir de 1946 que l'on peut commencer à évaluer les nouvelles voies offertes aux femmes et parler de l'émer-gence ou de la nonémergence d'une femme nouvelle.

4 La Constitution de la Quatrième République d'octobre 1946 accorde les mêmes droits aux hommes et aux femmes. Dans le préambule, il est stipulé que chacun a droit au travail ; le préambule promet aussi que la nation « assure à l'individu et à la famille les conditions nécessaires à leur développement ». De plus, en 1946, le décret Ambroise Croizat («A travail égal, salaire égal »), l'établissement des sections féminines dans les partis politiques, la mise en place progressive d'un système de sécurité sociale 
généralisée et d'un système d'allocations familiales portent à croire que l'ère de l'égalité est arrivée. Les luttes féministes sont dépassées, disent même certains groupes féministes. Or les années qui vont suivre accusent une confusion et une déception chez les femmes en général et chez les féministes en particulier. Cet article, tout en acceptant les profonds changements opérés par la Libération, considérera plutôt les ambiguïtés des discours et des politiques qui, me semble-t-il, ont fortement contribué à freiner l'émergence d'une femme nouvelle - et d'une nouvelle féminité - dans la France de l'après-guerre.

5 L'ambiguïté des discours publics sur la féminité est déjà perceptible dans les articles de la Constitution: les femmes sont traitées comme individues dans certains articles, comme mères de familles dans d'autres ${ }^{1}$. Un conflit éventuel entre le rôle maternel et le rôle individuel des femmes n'est même pas imaginé. D'autres contradictions s'y ajoutent. D'une part, la notion de nouveauté et le désir de mettre une distance entre la guerre et l'après-guerre sont très puissants; d'autre part, la guerre ayant représenté une interruption de la vie normale, le désir d'un « retour à la normale » est également puissant. La Libération, période où s'ouvre une ère nouvelle est le moment de grandes transformations pour les femmes; en même temps, la Libération, période d'ajustement dans la vie des couples, n'est peut-être pas le moment pour envisager la transformation des rôles sexués. Un cloisonnement imaginaire séparant public et privé permet à la contradiction entre le nouveau et la normale de passer inaperçue : le nouveau, c'est pour la vie publique; dans le privé, rien ne doit changer. Il s'agit, pour les femmes, d'être à la maison des femmes comme avant, et en même temps de chercher à être femme différemment dans la vie publique.

6 A la Libération, les partis politiques parlent des trois fonctions des femmes - mère, travailleuse, citoyenne ${ }^{2}$. De ces fonctions, celle de citoyenne est radicalement nouvelle et c'est dans le discours de la femme citoyenne (et même représentante) que l'on peut espérer voir surgir la femme nouvelle. La fonction de la femme travailleuse est celle qui démontre le plus d'ambiguïtés, notamment lorsque le travail professionnel est considéré en relation avec la maternité ; la fonction maternelle est celle qui domine les autres, qui contient au premier abord le moins d'équivoque et qui semble être le moins susceptible de changement.

Discours de la femme nouvelle : la citoyenne et la députée

7 C'est dans la vie publique que la femme nouvelle est censée prendre sa place. Cette femme nouvelle est accueillie avec enthousiasme par certains et réticence par d'autres. Elle est l'objet d'une inquiétude évidente. L'inquiétude principale semble être celle de savoir si une femme peut participer à la vie politique sans "perdre sa féminité » question posée sur les pages des journaux politiques et des magazines féminins de l'époque. Quelques exemples: Germaine Peyroles du MRP (" avocate, quatre enfants »), interviewée dans le quotidien $L^{\prime}$ Époque ${ }^{3}$ (9/4/46), dit :

aucun grand problème ne peut être étranger aux femmes. Mais la femme doit rester femme.

De même, dans le courrier des lectrices de Femmes Françaises, journal de l'Union des Femmes Françaises, du 12 octobre 1944, on peut lire l'opinion suivante :

Il est du devoir de chaque femme de s'intéresser à la vie politique du pays, sans pour cela changer sa féminité contre des allures qui feraient pâlir nos compagnons.

9 Féminité semble impliquer dépendance, stupidité, ruse. Ainsi, dans Elle (30/4/46), nous lisons : 
Alors, si les programmes politiques vous paraissent obscurs, si vous confondez MURF et MRP, UDSR et URD, faites confiance à votre mari. Peut-être que si vous le lui demandez gentiment, il poussera la complaisance jusqu'à vous expliquer les motifs de sa décision.

10 Ou dans La Femme, journal des femmes du Mouvement de Libération Nationale $(29 / 5 / 46)$ :

Vous avez bien raison d'être contente; vous avez senti que vous faisiez partie de quelque chose de grand, d'important et que ce bulletin de vote conférait à votre mince personne un peu de cette grandeur et de cette importance [...]. Dans l'ensemble, les femmes ont pris conscience de leur rôle de citoyenne sans nuire à leur qualité de femme. Si à la sortie du bureau de vote, vous arrangez une bouche ou maniez votre poudrier, votre mari sera rassuré...

Le même langage s'emploie lorsque les partis essayent d'entreprendre l'éducation politique des femmes, censées être fondamentalement apolitiques. Ainsi, La Vie Heureuse(4/12/46), magazine socialiste, écrit :

Vous savez, chères lectrices, ce que c'est qu'un budget. Vous faites vous-mêmes votre budget à l'intérieur de votre maison. Vous savez donc par expérience qu'avec les mêmes recettes les ménagères... obtiennent des résultats très différents... c'est la même en ce qui concerne le budget de la France.

12 Si la nouvelle citoyenne est une créature surtout simple et sans opinion politique, à qui il faut tout expliquer en termes domestiques, la nouvelle représentante (déléguée, députée, sénatrice) est une créature qui dérange. La presse politique et féminine cherche à rassurer le grand public en général et les femmes en particulier, qu'il est fort possible d'entrer dans la vie publique et de "rester femme». Une interview avec Germaine Poinso-Chapuis (députée MRP qui sera brièvement au gouvernement en 1947-48) la montre en photo, entourée de ses enfants, des chaussettes à raccommoder à portée de main; Marie-France (12/2/47) note, dans un article sur les femmes en politique, que Madame Lagarde, première femme à être nommée à la Cour de Cassation " assure, outre ses fonctions officielles, la direction d'un foyer où 5 enfants font sa joie et son cher souci ». On peut multiplier les exemples de ce type.

Le féminisme revendicatif disparaît des partis au bout de deux ou trois ans. Les paroles suivantes de Jeannette Vermeersch, prononcées devant les membres du PCF au Velodrome d'hiver le 11 avril 1945 font preuve d'un féminisme étonnant pour l'époque (et pour elle) et ne seront pas entendues une deuxième fois :

Des gens, peut-être bien intentionnés, prétendent que la place de la femme est au foyer. Peut-être ferait-on bien de consulter les femmes à ce sujet. Est-il tellement certain que les femmes considèrent que le rôle qu'elles auront à jouer éternellement dans la vie sera celui de cirer les souliers de la famille, de laver le linge ?... Non, la femme veut devenir un être libre et conscient et ne pas regarder la vie derrière le rideau de sa cuisine...

Deux ans plus tard, on n'entend plus ce genre de discours en France. Le seul groupe qui revendique l'étiquette « féministe » après la guerre est La Ligue Française pour le Droit des Femmes, dont la revue reparaît à partir de janvier 1946. Les rédactrices de la revue jettent un œil critique sur la vie publique, notent les paroles misogynes des journaux et des hommes politiques, citent les interventions des députées à l'Assemblée Nationale, applaudissent les succès féminins, organisent des cours d'instruction civique et politique et se battent pour la réforme des régimes matrimoniaux. Pourtant, l'influence de la Ligue, dont la plupart des adhérentes sont des femmes des milieux politiques radicaux, semble, malgré une réalité tout autre ${ }^{5}$, « proche du néant $»^{6}$. En référence à 
l'avant-guerre, "féminisme" semble indiquer suffragisme, mouvement bourgeois, femmes mondaines, et le terme est soigneusement évité par la plupart des groupes de femmes. Où est donc cette femme nouvelle?

Discours de la femme nouvelle : la travailleuse

Les femmes françaises ont toujours constitué un pourcentage relativement élevé de la population active. Pour elles, travailler n'est pas une nouveauté de l'après-guerre. La nouveauté réside dans le discours sur la femme travailleuse, d'une part, et dans l'entrée des femmes plus aisées et des femmes mariées sur le marché du travail, de l'autre. Les chiffres des recensements montrent déjà cette évolution qui deviendra plus claire dans les années 1960, mais qui s'amorce, occultée par l'exode rural et le déclin de la part du secteur primaire dans l'économie française, dans les années 1940 et $1950^{7}$. Ils révèlent un accroissement continu de la proportion de femmes mariées dans la population active féminine non-agricole entre 1946 et 1962 (de 39,1\% à 50\%) et aussi du taux d'activité des femmes mariées non-agricoles (de 19,6\% à 24,5\%). Le journal de l'Union Nationale des Associations Familiales, Pour la Vie, a bien noté en 1947 que «95\% de femmes ayant 9 enfants ou plus » restent chez elles ${ }^{9}$, mais celles ayant deux ou même trois enfants commencent à entrer sur le marché du travail professionnel. Le taux d'activité dépend, bien sûr, du nombre d'enfants par femme et de l'âge de ses enfants, mais aussi du niveau scolaire de la femme : plus elle est diplômée, plus elle travaille et reste au travail.

Les femmes diplômées, exerçant un métier bien rémunéré et agréable, sont pourtant bien peu nombreuses. La majorité se trouve toujours cantonnée dans les postes subalternes, sans qualification ni responsabilité, à bas salaire. De plus, l'opinion publique considère que l'idéal serait qu'un homme gagne un salaire permettant à sa femme de rester au foyer. Le travail féminin représente en effet un domaine de conflit par excellence, à la fois au niveau personnel et au niveau de la politique. Une politique du travail et une politique familiale qui, ensemble, rendent tolérable la vie des femmes qui cumulent travail et famille, ne sont pas envisagées. Dans les années qui suivent immédiatement la guerre, il n'y a simplement pas de politique du travail féminin, malgré le besoin de main d'œuvre. La natalité est la première préoccupation des gouvernements, et comment encourager à la fois la natalité et le travail féminin ? La contradiction entre les deux est flagrante, notée dans la presse. La Nouvelle République, journal à sympathie gaulliste, remarque, le 8 novembre 1946 :

il est certain qu'il y a là deux politiques qui s'opposent et qu'on ne saurait marquer la place de la femme à l'usine sans consentir en même temps à la désertion et au dépeuplement des foyers.

17 Les partis politiques en présence sont clairs: pour le MRP, le travail féminin est la catastrophe, le foyer vide, l'enfant abandonné et délinquant, un motif de divorce. Le journal du parti, Forces Nouvelles, écrit en décembre 1946 que «Le MRP est le mouvement qui affranchit la femme, pas pour la mettre à l'usine, mais pour la rendre à sa famille ». Ce refus du travail féminin se voit aussi dans le nouveau magazine MarieFrance, qui en commentant le Congrès de l'UfF écrit le 23 janvier 1946 :

Nous faudra-t-il aussi, nous les vraies femmes, tenir un congrès pour revendiquer un droit... le droit sacré pour les femmes d'être de vraies femmes, de ne pas être obligées de travailler au bureau ou à l'atelier quand elles veulent rester à leur place pour élever dignement les bons petits Français dont la Nation a besoin? 
Malgré la tendance reconnue irréversible de l'accroissement du taux d'activité nonagricole des femmes, les «vraies » femmes sont celles qui préfèrent rester au foyer, soutenues par l'allocation de salaire unique ${ }^{10}$. Comme le souligne Jean Daric,

La femme travailleuse est mieux acceptée si elle travaille par nécessité économique plutôt que par désir d'une certaine autonomie, et si son travail prend très clairement une place inférieure à son rôle domestique et familial. À quelques exceptions près, la femme travailleuse n'est jamais célébrée. Les conditions du travail féminin ne s'y prêtent guère : le travail féminin non agricole en France est caractérisé par une qualification peu élevée, et, par suite, par des salaires bas [...] le travail féminin demeure, pour le ménage, un travail d'appoint assez mal rétribué. ${ }^{11}$

Le PCF et la CGT évidemment évoquent les femmes travailleuses, notamment dans les publications de l'UFF et, à partir de 1952, dans Antoinette, magazine des femmes de la CGT. Mais, pour ces organisations ouvrières, le travail occupe une place paradoxale : d'une part il est source d'émancipation, de l'autre il est source d'oppression. Les discours sur le travail féminin reflètent cette ambiguïté et aussi les ambitions électorales du Parti qui ne veut pas aliéner l'électorat potentiel que représentent les femmes au foyer. Pour la CGT, le travail féminin est un fait concret et il faut essayer d'améliorer les conditions dans lesquelles les femmes travaillent. En 1948, la CGT établit une " commission femmes » et demande, sans avoir beaucoup de succès, une meilleure formation professionnelle pour les jeunes femmes ainsi qu'une extension du réseau de crèches et garderies. Tous les ans au congrès de la CGT, la section féminine réitère les mêmes demandes. La gauche politique et syndicale est hostile à l'introduction de l'allocation de salaire unique, mais elle ne la combat pas, par peur de faire perdre de l'argent aux familles qui en ont besoin. Elle dénonce également « l'idéologie bourgeoise de la femme au foyer ", le salaire d'appoint, mais en même temps elle demande la reconnaissance de la fonction maternelle comme fonction sociale ${ }^{12}$.

Dans les professions de haut niveau, la magistrature s'ouvre aux femmes ainsi que la nouvelle École Nationale d'Administration fondée en 1946. Pourtant, très peu de femmes cherchent une carrière au sommet de l'État : entre 1946 et 1956, seulement 18 femmes sont passées par les portes de l'ENA, pour 708 hommes $^{13}$. Il faut attendre l'après-68 pour voir une femme préfet, une femme dans l'Inspection des Finances, une femme dans les cabinets ministériels ou une femme ambassadrice. De plus, la loi reste souvent lettre morte - il n'y a tout simplement pas salaire égal pour travail égal (principe qu'il faut d'ailleurs répéter en 1950 et encore en 1972) - et la pratique ne reflète pas l'égalité de principe. Les seules explications possibles, outre les explications économiques des employeurs, sont celles de la persistance de l'idéologie traditionnelle des rôles féminins et masculins, et le refus (ou du moins la réticence) des hommes de céder ou de partager le pouvoir, à n'importe quel niveau que ce soit. Le rôle domestique et maternel de la femme, qui est à la fois une réalité et une codification discursive, influe sur ses possibilités professionnelles, pour les bloquer dans la plupart des cas.

Discours de la femme traditionnelle : la ménagère et la mère au foyer

21 Les rôles domestiques, la division du travail domestique et le partage du pouvoir domestique ne sont pas mis en question après la guerre. En 1945 une commission parlementaire chargée de considérer la révision du Code civil est établie, mais ses recomman-dations - s'il y en a eu - ne sont pas publiées. Entre 1945 et 1959, quinze propositions de loi sur le régime matrimonial sont avancées, mais aucune n'est déposée. Le Code civil maintient la femme mariée dans une situation de profonde inégalité financière et juridique par rapport à son mari. La situation ne sera 
(partiellement) rectifiée qu'en 1965. L'égalité théorique dans la vie publique ne trouve pas écho dans la vie privée.

Malgré cette inégalité de statut, les Françaises se marient encore plus nombreuses après la guerre, et connaissent la maternité en plus grand nombre aussi. Le destin de femme, épouse et mère, n'est pas contesté. Le discours du Général de Gaulle du 5 mars 1945, demandant aux Français « douze millions de beaux bébés pour la France » semble répondre aux désirs des Français, et le taux de natalité connaît un essor spectaculaire jusqu'en 1950, où les naissances se stabilisent autour du chiffre annuel de 800 000. L'on ne parle pas de planning familial ou de contrôle des naissances avant $1953^{14}$ et pendant le reste de la décennie, ils sont discutés seulement dans les milieux très restreints de l'association Maternité Heureuse, l'École des Parents et d'autres groupements progressistes ${ }^{15}$. Les lois de 1920 et de 1923, interdisant la propagande anticonceptionnelle et la pratique de l'avortement, sont toujours en vigueur et arrivent sur la scène politique seulement en 1965 dans la campagne présidentielle de François Mitterrand. La situation n'est modifiée qu'en 1967 (pour la contra-ception) et 1974 (pour l'avortement). À la Libération, la population française partage l'inquiétude de ses gouvernements successifs sur l'avenir démographique de la France, et la contribution féminine à la reconstruction doit être avant tout la reproduction. Les discours publics sur la question parlent en termes de "mission féminine » ou de " nature féminine » et aucune transformation remarquable n'intervient en ce qui concerne la fonction maternelle des femmes. La critique de la maternité, s'il y en a, porte sur les conditions dans lesquelles les femmes sont obligées d'élever leurs enfants, mais la maternité est toujours présentée comme le destin féminin ${ }^{16}$.

Il est sûr que la Libération apporte des changements dans la vie des femmes, pour l'améliorer dans la plupart des cas, mais ce que les gouvernements prévoient pour les femmes ne correspond pas toujours à leurs attentes. Si les Françaises cumulent maternité et travail professionnel, le pouvoir ne cherche pas à les aider et à concilier leurs rôles multiples. Elles sont, de plus, coincées entre les objectifs divers des partis politiques et des gouvernements successifs et ne constituent jamais l'objet d'une politique cohérente. Le monde politique et public demande aux femmes d'accomplir tant de rôles en même temps et le rôle maternel l'emporte sur le reste. Un discours qui envisage la maternité en d'autres termes ne viendra que plus tard - dans les années 1960 : celui de l'après-guerre est on ne peut plus traditionnel.

Peut-on donc parler d'une femme nouvelle? L'influence de la Résistance, si importante en 1945, disparaît assez rapidement. Les chiffres montrent un recul de la participation féminine à la vie politique. La politique familiale et sociale est surtout une politique nataliste. Les améliorations matérielles de la vie quotidienne et domestique rendent la vie au foyer plus accueillante que la vie professionnelle où la misogynie - ouverte ou couverte, consciente ou inconsciente - semble être à l'ordre du jour et rien n'est fait pour donner aux femmes l'impression de participer sur un pied d'égalité avec les hommes. La situation décrite dans cet article a tout pour décourager les femmes, pour les détourner de la vie publique et les cantonner dans la vie privée.

Pourtant, la Libération a posé les principes d'une évolution qui sera inévitable, même si elle n'est pas immédiatement visible ; la confusion qui règne sur la féminité et les rôles féminins freine l'entrée des femmes dans la vie publique mais, paradoxalement, porte aussi en elle les germes de cette évolution. Les choix faits par les femmes elles-mêmes, souvent en contradiction avec la politique et les discours dominants, y contribuent 
aussi de manière importante. Une femme nouvelle dans une France nouvelle? Il serait plus juste de parler d'une évolution lente et boiteuse, où la législation manque de cohérence, et où les discours et la pratique ne marchent pas du même pas. Toute la période de l'après-Libération est marquée par la confrontation entre la féminité traditionnelle et la recherche d'une féminité nouvelle, confrontation qui commence à s'apaiser seulement dans les années 1960. La déclaration de Mathilde Péri avait affiché un optimisme non confirmé par l'expérience des femmes sous la seule Quatrième République.

\section{NOTES}

1. Cf Jane Jenson, «The liberation and new rights for women ", in Behind the Lines, eds. Higonnet Margaret, Jane Jenson, Sonya Michel and Margaret Collins Weitz, Newhaven, Yale University Press, 1987 ; Claire Duchen, Women's Rights and Women's Lives in France 1944-1968, London, Routledge, 1994 ; Etre féministe en France. Contribution à l'étude des mouvements des femmes 1944-1967, Martine Muller, Danielle Tucat, Sylvie Van de Casteele-Schweitzer, Paris, IHTP, 1985. On attend la thèse de Sylvie Chaperon sur les mouvements de femmes entre 1945 et 1970.

2. Voir Etre féministe..., op. cit., et les publications du Parti Communiste Français et de l'Union des Femmes Françaises (Femmes Françaises, Heures Claires, Cahiers de l'UFF).

3. Ce quotidien de Paris, qui s'était sabordé en 1940, est partisan des idées gaullistes et hostile au gouvernement de gauche en 1946.

4. Mouvement qui unit les mouvements de résistance de la zone sud, notamment ceux qui sont déjà unis dans les MUR, et quelques petits groupes de la zone nord. La première rédactrice en chef du journal a été Lucie Aubrac.

5. Christine Bard, Les filles de Marianne. Histoire des féminismes 1914-1940, Paris, Fayard, 1995.

6. IHTP, op. cit., p. 12.

7. Françoise Guelaud-Leridon, Le travail des femme,s Cahier de l'INED n 42 , Paris, PUF, 1964 ; J-J. Carré, P. Dubois et E. Malinvaud, La Croissance française, Paris, Le Seuil, 1972 ; M. Guilbert et V. Isambert-Jamati, «La répartition par sexe », in Georges Friedmann et Pierre Naville eds., Traité du Sociologie du Travail, Paris, Armand Colin, 1962.

8. Chiffres tirés de S. Fernandez, Transformation de l'activité féminine : facteurs évolutifs, Dossier de recherche ${ }^{\circ}$ 6, Centre d'Etudes de l'emploi, Paris, 1982.

9. Pour la Vie n' 15 , novembre 1947, p. 6.

10. Ce genre de discours est celui tenu par le MRP et la centrale syndicale CFTC, diffusé au public féminin dans les magazines plutôt traditionnels comme Marie-France, Femme Aujourd'hui etc. Il est contesté par la gauche de manière assez tiède.

11. Jean Daric, « Le travail des femmes : professions, métiers, situations sociales et salaires ", in Population n ${ }^{\circ}$, oct-dec. 1955, p. 690.

12. Voir notamment La question féminine dans les congrès confédéraux et les conférences nationales de la CGT 1948-1982, Supplément aux Cahiers de l'Institut CGT d'histoire sociale (sd). 
13. Le Monde 23/3/57.

14. Date de l'article de la jeune doctoresse Marie-Andrée Lagroua Weill-Hallé dans une revue médicale La semaine des hôpitaux, beaucoup lu dans les milieux médicaux, mais systématiquement ignoré par la hiérarchie médicale. Voir Duchen, op. cit., pp. 123-5. 15. Voir Duchen, op. cit., chapitre 6.

16. Sauf, bien sûr, dans Le Deuxième Sexe publié en 1949...

\section{RÉSUMÉS}

Cet article considère l'expérience des femmes en France après la Libération, partant de la déclaration faite en 1945 qu'une «nouvelle femme » allait surgir de la guerre. L'article examine les trois rôles attribuées aux femmes (citoyenne, travailleuse et mère) et les trois discours qui s'y attachent. Ces discours contribuent à limiter les potentialités des femmes qui ne jouissent pas d'une participation à la vie publique à part entière. Les changements, qui deviendront visibles dans la vie des femmes plus tard, n'apparaissent pas dans les années de l'après-Libération, années où les femmes sont systématique-ment déçues par les mesures prises (ou non prises) à leur égard.

\section{Claire Duchen : New woman for a new France?}

This article considers the experiences of women in France after the Liberation, taking as a starting point the notion that a new woman might emerge from the war. The article discusses the three roles attributed to women (citizen, worker and mother) and explores the way in which the three discourses seemed to work to limit and circumscribe women's lives and potential. The changes that become visible in women's lives only appear later in the 1960s; the years of the liberation were years in which women's hopes were consistently disappointed.

\section{AUTEUR}

\section{CLAIRE DUCHEN}

Claire DUCHEN enseigne à l'École des études europennes à l'Université de Sussex en Angleterre. Elle a publié plusieurs ouvrages sur le féminisme et les femmes en France, notamment Feminism in France from May ‘68 to Mitterrand (Routledge 1986) et Women's Rigths and Women's Lives in France, 1944-1968 (1994). Elle fait partie du groupe éditorial de la revue féministe Women's Studies International Forum. 